\title{
Image Dehazing of Images Captured in Real-World Weather Conditions using Derived Guided Filter
}

\author{
Mayuri Madake \\ Department of Computer Engineering \\ Marathwada Mitra Mandal's College of \\ Engineering, Pune
}

\author{
Sankirti Shiravale \\ Department of Computer Engineering \\ Marathwada Mitra Mandal's College of \\ Engineering, Pune
}

\begin{abstract}
Haze formation is the combination of airlight and attenuation. Attenuation decreases the contrast and airlight increases the whiteness in the scene. Atmospheric conditions created by floating particles such as fog and haze, severally degrade image quality. Removing haze from single image of a weather degraded scene found to be a difficult task because the haze is dependent on the unknown depth information, haze removal algorithms become more beneficial for many vision applications such as surveillance system, object detection, tracking and segmentation.

This work focuses on removing block artifacts and degradation factors from a natural scene image containing fog, haze such that the enhancement of that image becomes very easy. In this paper Derived Guided Filter based visibility restoration approach will be used in order to solve the inadequate estimation of transmission map and color cast problem. The Dark Channel Prior along with derived Guided Filter is selected with an aim to apply techniques such as denoising, color correction, and implementing other forms of enhancement in a single image dehazing system.
\end{abstract}

\section{Keywords}

Dark Channel Prior, Derived Guided Filter, Dehazing, Fog, Haze, Transmission Map

\section{INTRODUCTION}

Different weather conditions such as haze, fog, smoke, rain, or snow will cause complex visual effects of threedimensional or chronological domains in images. Such effects may significantly degrade the performances of outdoor vision systems depend on image feature extraction or visual attention modeling, such as event detection, object detection, tracking, and recognition, scene analysis and classification, image indexing and retrieval. Removal of weather effects has recently received much attention, such as removals of haze, rain, and snow from image. Based on the fact that the amount of scattering depends on the distance of the scene points from the camera, the degradation is spatially variant. Based on the fact that haze is dependent on the unknown depth, dehazing is therefore a challenging problem. If the available input is only one single hazy image, the problem is under-constrained. Hence, all of the old dehazing approaches have been proposed by using multiple images or additional information. Polarization- based methods were proposed to remove the haze effects through two or more images taken with different degrees of polarization. More constraints obtained from multiple images of the same scene under different weather conditions were employed for haze/weather effect removal. Moreover, in depth-based methods, it is required to provide some depth information from user inputs or known 3D models for dehazing or de weathering. Nevertheless, taking multiple input images of the same scene is usually impractical in several real applications. Single image haze removal has recently received much attention. The success of these approaches usually lies on using stronger priors or assumptions. It is observed that a haze-free image should have higher contrast compared with its hazy version.

Hence it is preferred to design a new single image haze removal algorithm to sidestep those problems publicized above. In this paper, a new single image haze removal algorithm is proposed by leading a minimal color channel and a atmospheric light term. The minimal color channel of a pixel is defined as the minimal value among all color components of the pixel. A simplified dark channel is calculated by using the minimal color channel. The preliminary value of the transmission map is assessed by using the simplified dark channel. The map is then cultured via derived guided filter [4] and is finally used to recover the haze image. The new haze removal algorithm can avoid/reduce halo artifacts, noise in the sky, and color alteration from appearing in the dehazed image. In addition, a very small amount of haze is left for the distant objects by the proposed haze removal algorithm. As a result, the sensitivity of depth in the dehazed image could be conserved better $[2,16]$.

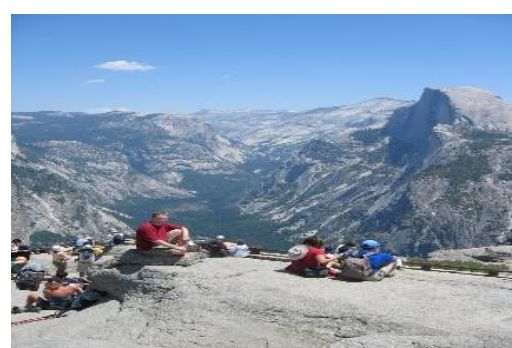

Fig: 1 Input Hazy Image

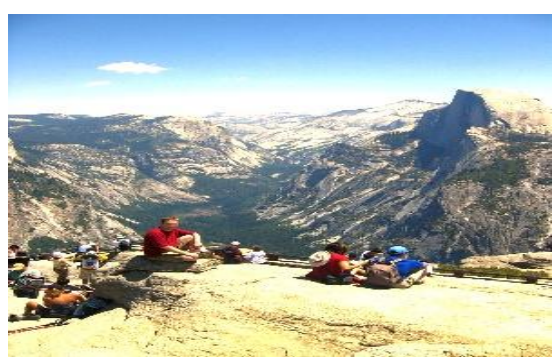

Fig:2 Enhanced Dehazed Image

The rest of this paper is organized as follows. Existing works on dehazing summarized in Section 2. Section 3 includes details on the recommended haze removal algorithm. Wide investigational results are given in Section 4 to illustrate the efficacy of the proposed algorithm. Concluding remarks are provided in Section 5. 


\section{SURVEY ON IMAGE DEHAZING}

Bad weather condition such as haze, mist, fog and smoke degrade the quality of the outdoor scene. It is an annoying problem to photographers as it changes the colors and reduces the contrast of daily photos, it diminishes the visibility of the scenes and it is a threat to the reliability of many applications like outdoor surveillance, object detection, it also decreases the clarity of the satellite images and underwater images. So removing haze from images is an imperative and broadly demanded area in computer vision and computer graphics. The image quality of outdoor scene in the haze, fog, mist and other bad weather condition is usually degraded by the scattering of a light before reaching the camera due to these large quantities of suspended particles (e.g .fog, haze, smoke, impurities) in the atmosphere. This phenomenon affects the normal work of automatic monitoring system, outdoor recognition system, tracking and segmentation intelligent transportation system. Scattering is caused by two fundamental phenomena such as attenuation and airlight. Haze attenuates the light reflected from the scenes, and further blends it with some additive light in the atmosphere. The target of haze removal is to improve the reflected light (i.e., the scene colors) from the mixed light. The constancy and strength of the visual system can improve by the usage of effective haze removal of image. There are many methods available to remove haze from image like polarization, independent component analysis, dark channel prior etc.

Tan and Oakley [15], Narasimhan et al. [11], and Kopf et al. [8] proposed haze removal approaches based on the given depth information. This information is gained from additional operations and interactions, such as assuming the altitude, tilt, position of the camera [15], or through manual approximation of the distance distribution of the sky area and invisible point in a captured image [11], or through an approximate 3D geometrical model of the captured scene [8]. The information of scene depth must be provided through user interaction, though it is probably given for an arbitrary image. So, these methods do not use for real time application in arbitrary images. Haze removal techniques go to the multiple image approaches category needs two or more images to estimate scene depth and remove haze formation. Schechner et al. [12], [7] proposed a method which uses multiple images of the same scene with different polarization degrees produced by rotation of a polarizing filter to compute scene depth and recover the true color of captured images. Methods proposed by Narasimhan et al. [13][11] estimate scene depth and then remove haze by comparing two images that are captured under different weather conditions. However, the above haze removal methods using multiple images usually require extra cost or hardware in order to perform effectively. Recently, research has aimed to single image haze removal techniques which use strong assumptions or priors [13] [2]. Tan [13] proposed a method which restores hazy images via a single input image by maximizing the local contrast of the restored image based on an observation that haze-free images possess higher contrast than input hazy images. This method can produce better results, but restored images may contain some block artifacts near depth discontinuities. The approach of Fattal [10] removes the haze by estimating the percentage of radiance of a scene and deducing the transmission medium from a single input image based on the assumption that transmission and surface shading are locally uncorrelated. However, this method [10] can fail when input images contain thick haze. He et al. [5] proposed a method which uses a assumption that most local patches for outdoor haze-free images exhibit very low intensity in at least one of color channel, which can be used to directly estimate haze density and recover true colors. Currently, the method of $\mathrm{He}$ et al. [5] is considered to be the best single image haze removal approach. However, the effect of haze removal method may change in response to various scene objects in real environment. In particular, but the method proposed by He et al. [5] cannot deal with complex structures. In such situations, restored images will feature block artifact. This is because transmission in a local patch is assumed to be constant; the recovered images in the complex areas will still contain halo effects near the complicated edge-structures even when transmission is optimized by the soft matting technique. Haze removal algorithms become more useful for many vision applications. It is found that most of the existing researchers have neglected many issues; i.e. no technique is accurate for different kind of circumstances. The survey has shown that the presented methods have neglected the techniques to reduce the noise issue which is presented in the output images of the existing fog removal algorithms. The problem of uneven and over illumination is also an issue for dehazing methods.

\section{PROPOSED WORK}

Proposed method which indicating the two modules. First module is based on dark channel prior technique which is used to directly estimate the transmission map of hazy image and the atmospheric light. Second module integrates the results obtained from first module to restore visibility of input hazy image. It is established that the proposed method is more appropriate for obtaining the better quality of the image than the most of the existing methods. The results made by the existing dark channel prior method have less PSNR value and more MSE. Therefore, the overall objective is to improve the results by coalescing derived guided filter with dark channel prior method. The proposed algorithm designed and implemented in python using opencv libraries. The detail algorithm for proposed system is given below.

Step 1: Pass the input hazy image I to the system

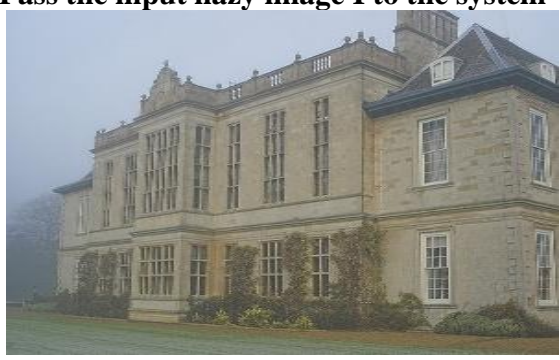

Fig:3 Input Hazy Image

Step 2: Calculate Minimum Dark Channel of input hazy image.

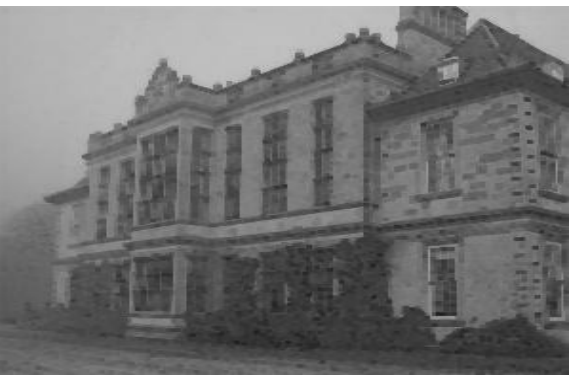

Fig:4 Minimum Dark Channel.

$\mathrm{b}, \mathrm{g}, \mathrm{r}=\mathrm{cv} 2 . \operatorname{split}(\mathrm{I})$

$\mathrm{dc}=\mathrm{cv} 2 \cdot \min (\mathrm{cv} 2 \cdot \min (\mathrm{r}, \mathrm{g}), \mathrm{b})$ 
Where I is the input image and $\mathrm{b}, \mathrm{g}, \mathrm{r}$ are the blue, green, red color channel [5].

\section{Step 3: Atmospheric light estimation}

For estimation of atmospheric light pick the top $0.1 \%$ brightest pixels in the dark channel. Among these pixels, the pixels with highest intensity in the input image I is selected as the atmospheric light [5].

\section{Step 4: Estimation of transmission map

$$
\mathrm{F}(\mathrm{t})=1-\omega^{*} \mathrm{dc}
$$

Where $\mathrm{F}(\mathrm{t})$ is function derived from obtained minimum dark channel dc and $\omega$.

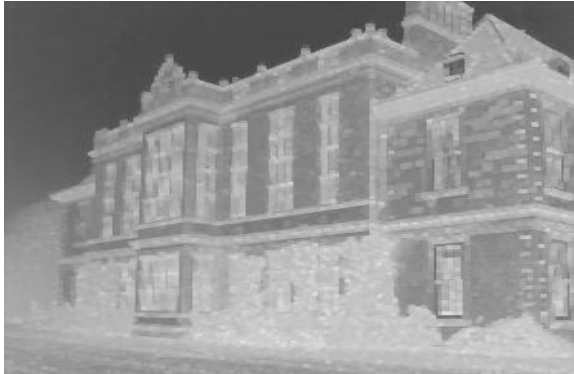

Fig:5Estimated Transmission Map.

In general, even in clear days the atmosphere is not totally free of any particle. So, the haze still exists when distant objects are referred. If the haze is removed comprehensively, the image may seem unusual and the sensation of depth may lose. So its optionally to keep a very small amount of haze for the distant objects by hosting a constant parameter $\omega(0<\omega \leq 1)$ into algorithm.

As per [3] $\omega$ have set value to 0.95 .

Step 5: Refinement of transmission map using derived guided filter.

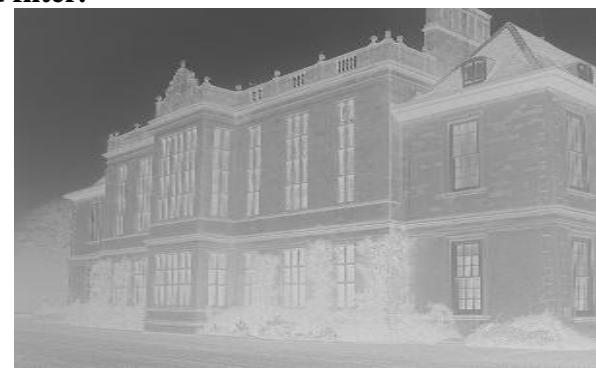

Fig:6 Refined Transmission Map.

For the refinement of transmission map, the filter is derived using existing BoxFilter named as derived guided filter [4].

BoxFilter is basically an average of surrounding pixel. it is actually a convolution filter which is commonly used mathematical operation for image filtering. A convolution filters provide a method of multiplying two arrays to produce a third one. In box filtering, image sample and the filter kernel are multiplied to get the filtering results. The algorithm for derived guided filter is written as below:

$\mathrm{r}=8$;

$€=0.0001$;

mean_I =cv2.boxFilter(g, cv2.CV_64F,(r,r));

mean_p=cv2.boxFilter(tr, cv2.CV_64F,(r,r));

mean_Ip $=$ cv2.boxFilter $(\mathrm{g} *$ tr, cv2.CV_64F, $(\mathrm{r}, \mathrm{r}))$;

cov_Ip $=$ mean_Ip - mean_I*mean_p;

mean_II = cv2.boxFilter $\left(\mathrm{g} * \mathrm{~g}, \mathrm{cv} 2 . \mathrm{CV} \_64 \mathrm{~F},(\mathrm{r}, \mathrm{r})\right)$;

var_I $=$ mean_II - mean_I*mean_I; $\mathrm{a}=$ cov_Ip/ $($ var_I $+€)$;

$\mathrm{b}=$ mean_p - a*mean_I;

mean_a $=$ cv2.boxFilter (a, cv2.CV_64F, $(r, r))$;

mean_b = cv2.boxFilter (b, cv2.CV_64F, $(r, r))$;

$\mathrm{t}=$ mean $\_\mathrm{a}^{*} \mathrm{~g}+$ mean $\_$;

In this algorithm, mean is a mean filter with a window radius r. The abbreviations of variance (var), and covariance (cov) indicate the intuitive meaning of these variables.

Where filtering input image is transmission map tr, guidance image will be gray image $\mathrm{g}$ obtained from input image, radius $\mathrm{r}$, regularization $€$ and $\mathrm{t}$ is obtained refined transmission map.

Step 6: Visibility Enhancement of hazy image.

$$
\mathrm{F}(\mathrm{v})=(\mathrm{I}-\mathrm{A} / \max (\mathrm{t}(\mathrm{x}), \mathrm{t} 0))+\mathrm{A}
$$

Where $F(v)$ is the visibility enhancement function A - Estimated atmospheric light

I - Input Image

$\mathrm{t}(\mathrm{x})$ - transmission map and $\mathrm{t} 0=0.1$ to restrict the transmission $t(x)$ to a lower bound $t 0$, which means that a small certain amount of haze is preserved in very dense haze regions [3]

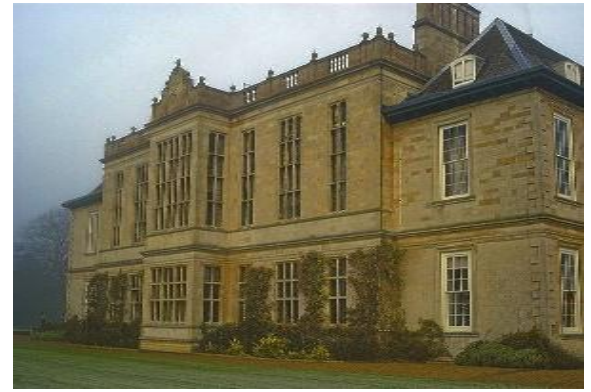

Fig:7 Output Dehazed Image.

\section{EXPERIMENTAL RESULTS}

In this section comparison between the results of the images by the prevailing method implemented using laplacian filter [2] and the proposed method will be done. The results of the existing and the proposed approaches are shown as below.

Table 1. Shows qualitative comparison between proposed and existing method. Column A, B, C shows Input hazy image, results of existing method and result of proposed method respectively.
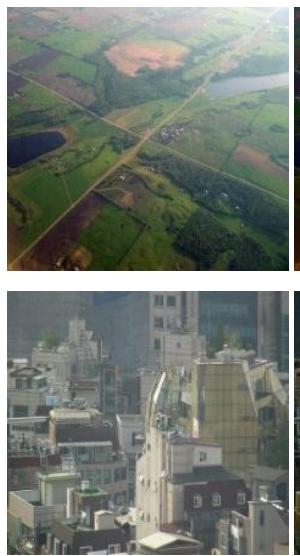
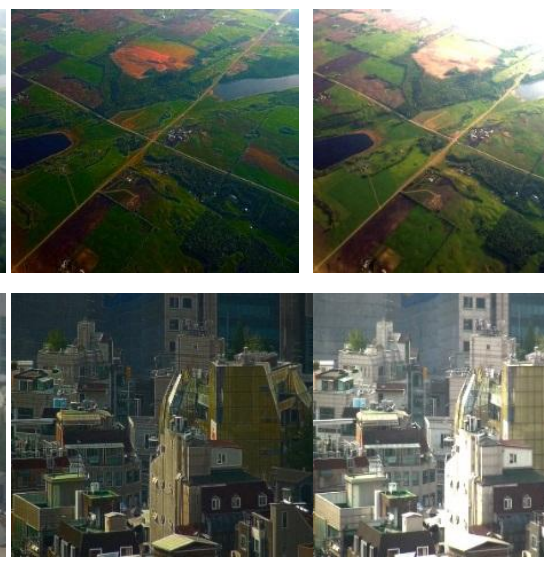

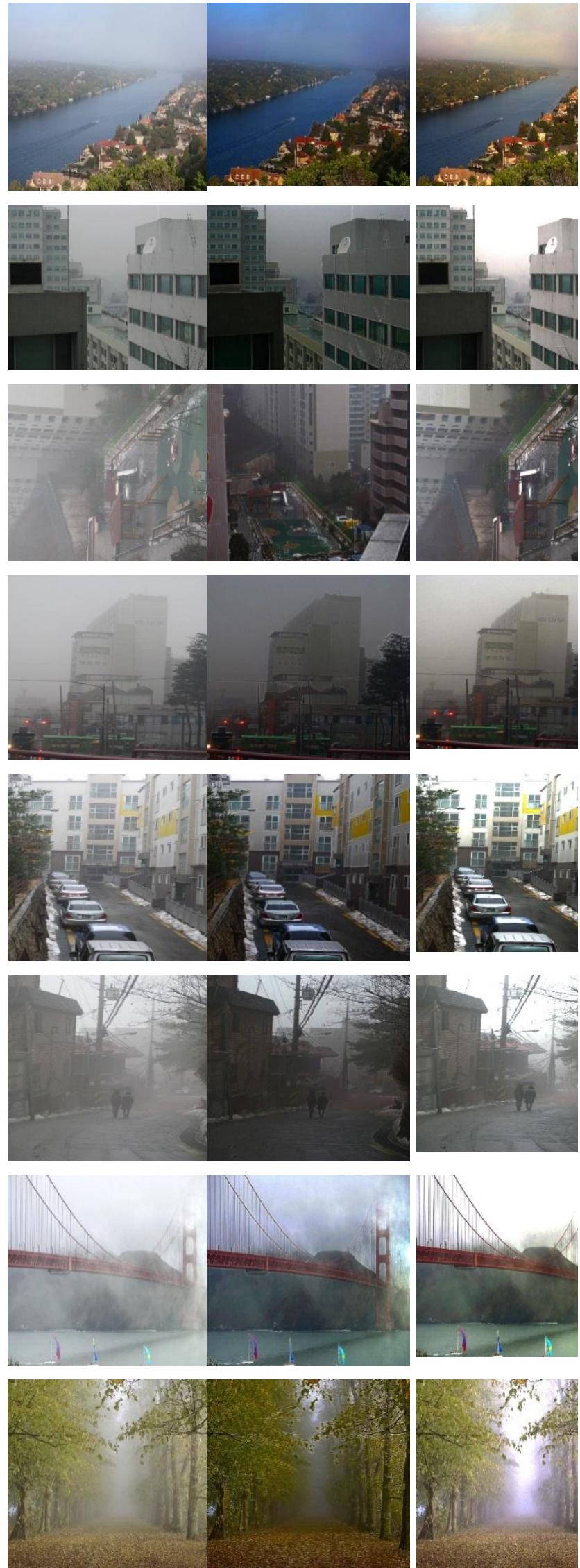
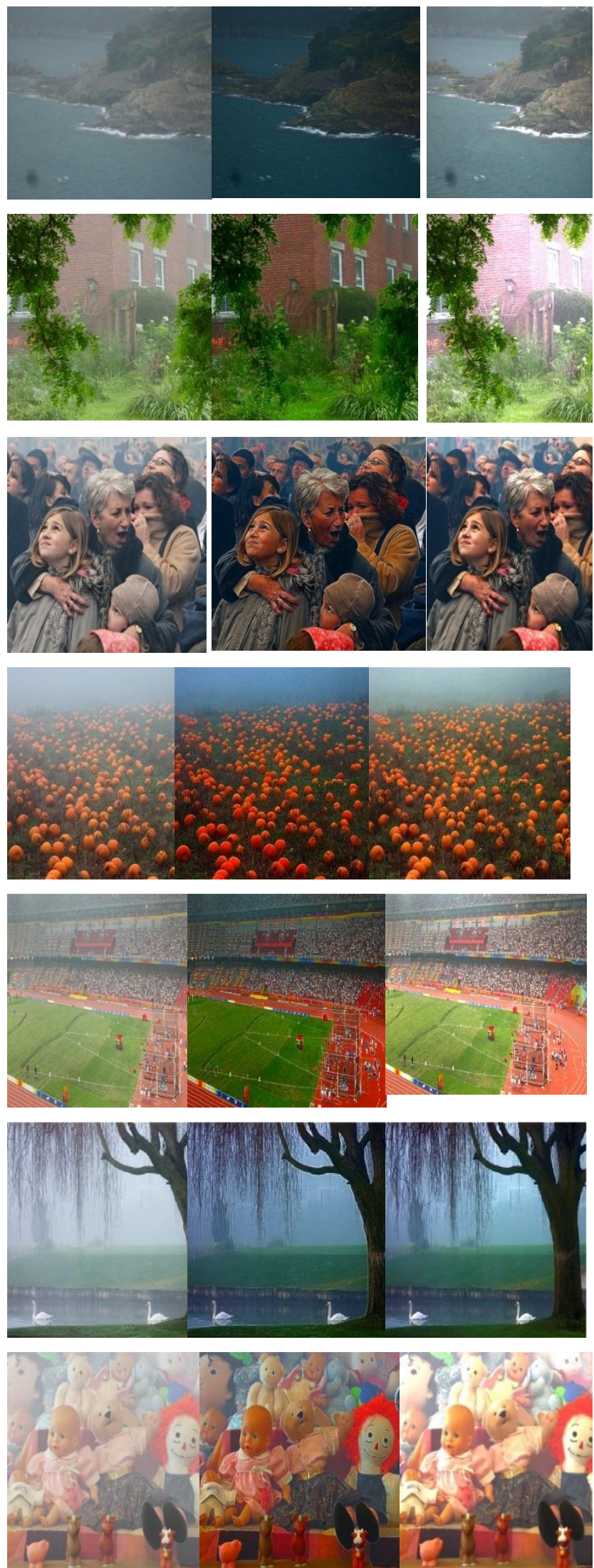


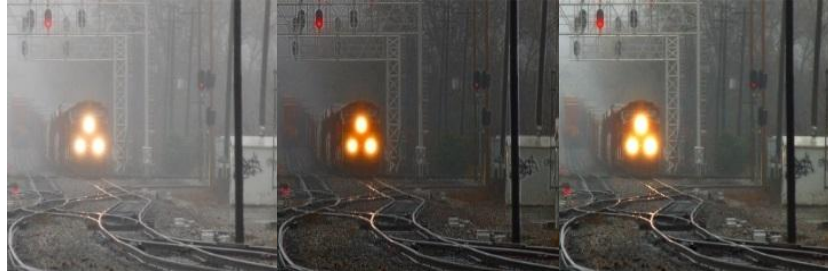

\section{PERFORMANCE ANALYSIS}

The proposed algorithm is tested on different types of hazy images. The algorithm is applied using some performance indices peak signal to noise ratio (PSNR), Mean squared error (MSE), visible edge restoration rate (V) and Required dehazing time. Implementation of the proposed algorithm has been done using python and Opencv libraries in Spyder 24.0.0 editor. The developed approach is compared against a well-known image dehazing technique available in literature [2] that is using laplacian filter. Here is the comparison of proposed approach using some performance metrics. Result shows that oproposed approach gives better results than the existing technique.

Fig 8 is showing the quantized analysis of the mean square error. As MSE need to be reduced, the proposed algorithm is showing the better results than the available methods as mean square error is less in every case. The mean Square error is reduced in each case than the existing method. The proposed method is tested on the number of images and in each case shows the better results than the existing method.

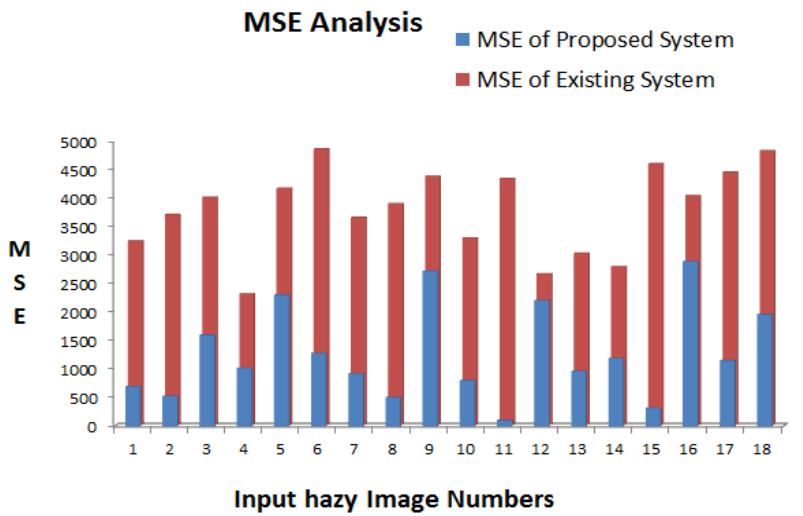

Fig:8 Mean Square Error Analysis

Fig 9 is showing the comparative analysis of the Peak Signal to Noise Ratio (PSNR). As PSNR need to be maximized; so the main goal is to rise the PSNR than existing method. Fig 9 has clearly shown that the PSNR has been increased in the case of the proposed algorithm; therefore, proposed algorithm is providing better results than the existing methods. The proposed technique is tested on the number of images and in each case shows the better results than the existing method.

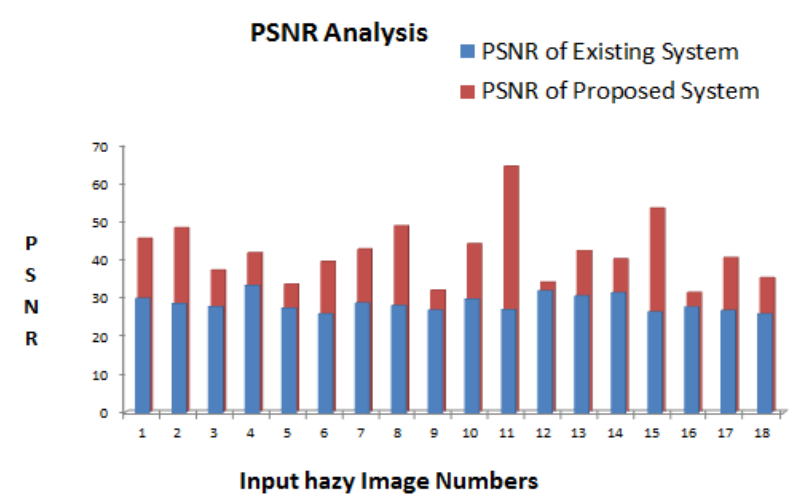

Fig: 9 Peak Signal to Noise Ratio Analysis.

Fig 10 is showing the comparative analysis of the Visible edge restoration rate [3]. As per analysis the rate need to be maximized; so the more visible edges should get restore Fig 10 has clearly shown that the rate has been increased in the case of the proposed algorithm; therefore, proposed algorithm is providing better results than the existing methods.

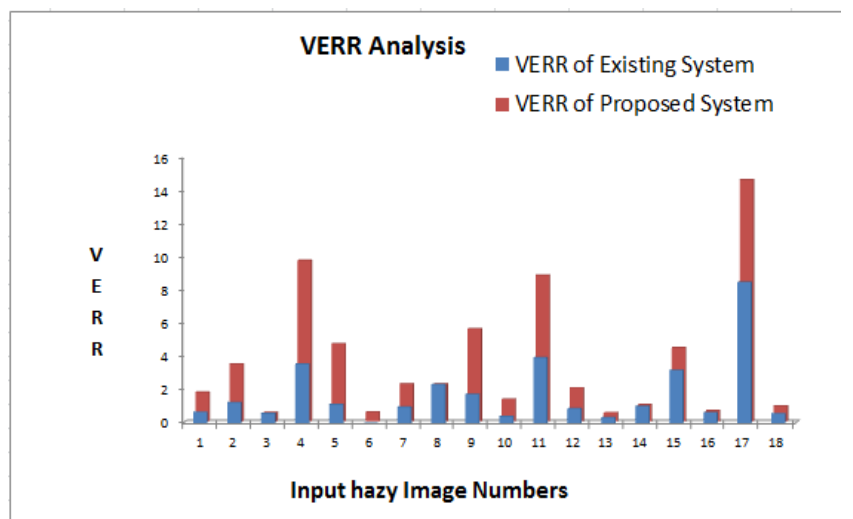

Fig:10 Visible Edge Restoration Rate Analysis.

Fig.11 is showing the comparative analysis of the time required to dehazing of image by both systems. According to graph the dehazing time is decrease comparatively with existing system, which tends to faster dehazing of image.

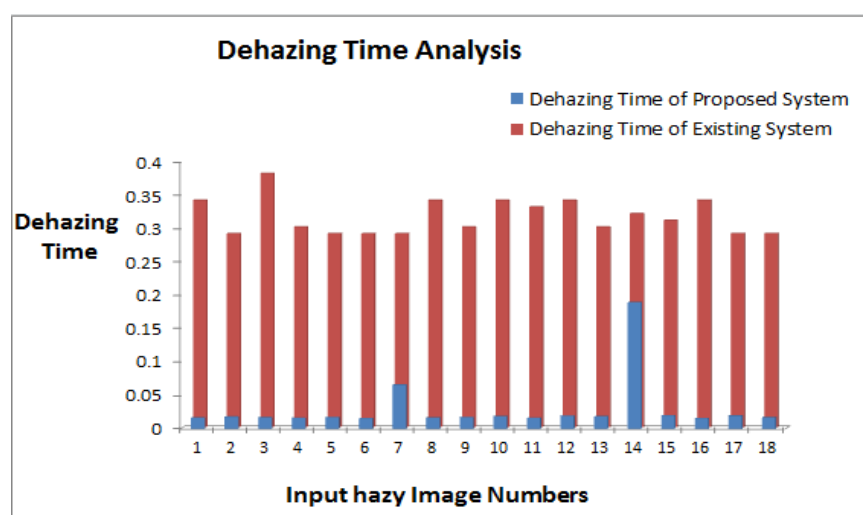

Fig:11 Required Dehazing Time Analysis.

\section{CONCLUSION}

This research present a removing hazes from given hazy image. The proposed idea of enhancing visibility of hazy image using derived guided filter along with dark channel prior is presented in this paper. By using this method, the problem of dehazing can be solved without generating block 
artifacts and underexposed scene images, also it resulted in better edge preservation in comparison with existing method. The proposed algorithm implementation is done using Python including Opencv libraries which was tested on more than 100 different natural hazy images for analysis. As per analysis the proposed method gives better results than the existing one, which has been proven using different standard parameters listed above

Future work will refer to application of this method in real time systems like Surveillance system, object tracking, Driving guidance system, object detection technique etc..

\section{ACKNOWLEDGMENTS}

We would like to thanks Department of Computer, MMCOE, for support and encouragement for this project

\section{REFERENCES}

[1] Wencheng Wang et.al, "Fast Image Dehazing Methods using Linear Transformation", IEEE Transactions on Multimedia ( Volume: 19, Issue: 6, June 2017 )

[2] Shih-Chia Huang et.al. "An Advanced Single-Image Visibility Restoration Algorithm for Real -World Hazy Scenes", IEEE Transactions on Industrial Electronics, Vol. 62, No5, May2015.

[3] Shih-Chia Huang et.al., "Visibility restoration of single hazy images captured in real-world weather conditions", IEEE Transactions on circuits and systems for video technology, Vol 24, No. 10, Year 2014.

[4] Kaiming He et.al., "Guided Image Filtering" IEEE Transactions On Pattern Analysis and Machine Intelligence, Vol. 35, No. X, xxxxxxx 2013.

[5] Kaiming He et.al. "Single Image Haze Removal Using Dark Channel Prior", IEEE Transaction on Pattern Analysis and Machine Intelligence, Vol 38, No. 12. Year 2011.

[6] Ramandeep Kaur, Nitika Kapoor, "A Review on enhancement on foggy images", International Journal of Advances in Science and Technology (IJAST), Year: 2011
[7] E. Namer, S. Shwartz,Y.Y. Schechner, "Skylesspolarimetric calibration and visibility enhancement", Optics Expres., vol. 17, no. 2, pp. 472493,2009 .

[8] J. Kopf, B. Neubert, B. Chen, M. Cohen, D. Cohen-Or, O. Deussen, M. Uyttendaele, and D. Lischinski, "Deep Photo: Model-Based Photograph Enhancement and Viewing", ACM Trans. Graphics., Vol. 27, No. 5, pp. 116:1-116:10, Dec. 2008.

[9] R. Tan, "Visibility in Bad Weather from a Single Image", Proc. IEEE Conf. Computer Vision and Pattern Recognition, Jun. 2008.

[10] R. Fattal, "Single Image Dehazing", Proc. ACM SIGGRAPH08,2008.

[11] S.G. Narasimhan, and S.K. Nayar, "Interactive (De)Weathering of an Image using Physical Models, ICCV Workshop on Color and Photometric Methods in Computer Vision., pp. 1387-1394 Oct, 2003.

[12] Y.Y. Schechner, S.G. Narasimhan, and S.K. Nayar, Polarization Based Vision Through Haze, Proc. Applied Optics, special issue: light and color in the open air, vol. 42, no. no. 3, Jan. 2003.

13] S.G. Narasimhan, and S.K. Nayar, "Contrast Restoration of Weather Degraded Images", IEEE Trans. Pattern Anal. Machine Intell., vol. 25, no. 6, pp. 713-724, Jun. 2003.

[14] S.G. Narasimhan and S.K. Nayar, "Removing Weather Effects from Monochrome Images", Proc. IEEE Conf. Computer Vision and Pattern Recognition, 2001.

[15] K. Tan, and J. P. Oakley, "Enhancement of Color Images in Poor Visibility Conditions, Proc. IEEE Int. Conf. Image. Proc. (ICIP2000), Vol. 2, pp. 788-791, Sep. 2000.

[16] S.K. Nayar, and S.G. Narasimhan, "Vision in Bad Weather", Proc.Seventh IEEE Intl Conf. Computer Vision, Vol. 2, pp. 820-827, Jun. 1999. 